\title{
Prevalence of 22q11 microdeletions in DiGeorge and velocardiofacial syndromes: implications for genetic counselling and prenatal diagnosis
}

Deborah A Driscoll, Joshua Salvin, Beatrice Sellinger, Marcia L Budarf, Donna M McDonald-McGinn, Elaine H Zackai, Beverly S Emanuel

\begin{abstract}
Deletions of chromosome 22q11 have been seen in association with DiGeorge syndrome (DGS) and velocardiofacial syndrome (VCFS). In the present study, we analysed samples from 76 patients referred with a diagnosis of either DGS or VCFS to determine the prevalence of 22q11 deletions in these disorders. Using probes and cosmids from the DiGeorge critical region (DGCR), deletions of 22 q11 were detected in $83 \%$ of DGS and $68 \%$ of VCFS patients by DNA dosage analysis, fluorescence in situ hybridisation, or by both methods. Combined with our previously reported patients, deletions have been detected in $88 \%$ of DGS and $76 \%$ of VCFS patients. The results of prenatal testing for 22q11 deletions by FISH in two pregnancies are presented. We conclude that FISH is an efficient and direct method for the detection of 22q11 deletions in subjects with features of DGS and VCFS as well as in pregnancies at high risk for a deletion.

( $\mathcal{F}$ Med Genet 1993;30:813-17)
\end{abstract}

DiGeorge syndrome (DGS), a developmental field defect of the third and fourth pharyngeal pouches, is characterised by thymic aplasia or hypoplasia, absent or hypoplastic parathyroid glands, and conotruncal cardiac malformations. The aetiology is presumed to be heterogeneous. There have been reported cases of autosomal dominant, autosomal recessive, and $\mathrm{X}$ linked inheritance. ${ }^{1}$ Chromosomal abnormalities are not uncommon in patients with DGS. The majority of cytogenetically abnormal DGS patients initially reported had unbalanced translocations with monosomy 22pter $\rightarrow \mathrm{q} 11 .^{2-11}$ More recently, using high resolution banding techniques, visible interstitial deletions of 22q11.2 (del(22)(q11.21q11.23)) have been identified in approximately $25 \%$ of DGS patients. ${ }^{12}$ On the basis of the cytogenetic abnormalities, it was hypothesised that the DiGeorge critical region (DGCR) lies within 22q11. ${ }^{1314}$ Molecular studies have borne out this suggestion as we and others have shown that cytogenetically normal DGS patients have microdeletions of 22q11..$^{15-18}$ Our initial study began to delineate the DGCR. This region, bounded by locus D22S75 (N25) proximally and D22S259 (pR32) distally, was consistently deleted in the first 14 DGS patients we studied. ${ }^{15}$

More recently, we and others have shown that patients with velocardiofacial syndrome (VCFS) also have deletions of this same region of 22q11..$^{190}$ VCFS is an autosomal dominant disorder characterised by palatal abnormalities, cardiac defects, learning disabilities, and a typical facial appearance..$^{21-23}$ The presence of features common to DGS, such as neonatal hypocalcaemia and decreased lymphoid tissue, in some patients with VCFS suggested that these two disorders might share a common aetiology and pathogenesis. ${ }^{24}$ Cytogenetic studies using high resolution banding techniques detected interstitial deletions of 22q11.2 in approximately $20 \%$ of VCFS patients. ${ }^{19}$ Molecular studies using either restriction fragment length polymorphisms (RFLP) or dosage analysis detected 22q11 microdeletions in the majority of patients reported by our group and others. ${ }^{18-20}$

Since our initial reports we have evaluated an additional 36 patients presumed to have DGS and 40 patients with the suspected diagnosis of VCFS to determine the prevalence of 22q11 deletions in association with these disorders. In addition, we have applied a fluorescence in situ hybridisation (FISH) assay using cosmids from the DiGeorge critical region (DGCR) to detect 22q11 deletions directly in patients as well as in pregnancies at risk for a deletion. In this report we describe the results of DNA dosage and FISH studies, discuss the implications for genetic counselling, and describe the application of FISH for the prenatal diagnosis of 22q11 microdeletion syndromes.

\section{Materials and methods}

PATIENTS

Thirty-six patients with the presumed diagnosis of DGS and four parents of DGS patients with a 22q11 deletion were referred to our laboratory for molecular analysis. Forty patients with suspected VCFS were also analysed for evidence of a 22q11 deletion. Two cases were studied for prenatal diagnosis. Case 1. One of our previously reported, deletion positive VCFS patients (VCF-10) ${ }^{19}$ elected to have an amniocentesis during her second pregnancy. Amniocentesis was performed at 16 weeks' gestation. Cultured amniocytes were obtained for DNA analysis as well as FISH. Case 2. The normal parents of a deletion positive VCFS patient requested that FISH studies be performed to exclude a 22q11 deletion in a subsequent pregnancy. This study was performed as an adjunct to routine cytogenetic studies performed for advanced maternal 
age. Cultured chorionic villi were sent to our laboratory for analysis.

DNA ANALYSIS

DNA was extracted from lymphoblastoid or fibroblast cell lines and cultured amniocytes by routine methods. Southern blot analysis for dosage studies was performed as previously described. ${ }^{15}$ Probes N25 (D22S75) and R32 (D22S259), the most proximal and distal markers delineating the DGCR, respectively, were used in this study. Quantitative hybridisation of genomic DNA from the patients and a normal control was performed in triplicate to determine copy number, as previously described. ${ }^{15}$

FLUORESCENCE IN SITU HYBRIDISATION (FISH) Cosmids specific to loci D22S75 and D22S259 were isolated from a flow sorted chromosome 22 cosmid library (kindly provided by $P$ deJong). Cosmid cos 82 which maps to the long arm of chromosome 22 was used as a control probe (kindly provided by $\mathrm{H}$ Vissing). Metaphase spreads prepared from lymphoblastoid cell lines, peripheral blood lymphocytes, cultured amniocytes, or chorionic villi were cohybridised with biotinylated-11-d-UTP labelled test probes and the control probe, $\cos 82$, and visualised with fluoresceinated avidin. Twenty-five metaphase spreads were scored for copy number at each test locus.

\section{Results}

DIGEORGE SYNDROME

In the present study, 30 of 36 patients referred with the diagnosis of DGS were hemizygous at locus D22S75 (N25) or D22S259 (R32) or both by either dosage analysis, FISH, or by a combination of both methods (table). Dosage analysis identified 22q11 deletions in 19 patients. FISH detected a 22q11 deletion in nine patients. Two patients appeared deleted by both dosage and FISH. Six patients, studied by dosage analysis, did not appear to be deleted at either locus D22S75 or D22S259.

\section{VELOCARDIOFACIAL SYNDROME}

Microdeletions of $22 \mathrm{q} 11$ were detected in 27 of the 40 patients referred with the diagnosis of VCFS (table). Hemizygosity at one or both loci, D22S75 and D22S259, was shown in 12 patients by dosage analysis, seven by FISH, and eight by a combination of dosage and FISH. Thirteen patients suspected of having VCFS did not appear to have a deletion, seven

Detection of $22 q 11$ microdeletions by dosage analysis and FISH of DGS and VCFS patients.

\begin{tabular}{lcc}
\hline & \multicolumn{2}{c}{ Deletions detected/number studied (\%) } \\
\cline { 2 - 3 } Method applied & DGS & VCFS \\
\hline Dosage & $19 / 25$ & $12 / 19$ \\
FISH & $9 / 9$ & $7 / 10$ \\
Dosage + FISH & $2 / 2$ & $8 / 11$ \\
Total & $30 / 36(83 \%)$ & $27 / 40(68 \%)$ \\
\hline
\end{tabular}

by dosage, three by FISH, and three by both dosage and FISH.

\section{FAMILY STUDIES}

Molecular analysis of the parents of five probands, four with DGS and one with VCFS, showed a 22q11 deletion in one parent in each case (fig $1 \mathrm{~A}-\mathrm{E}$ ). One of these parents was hemizygous at D22S75 and D22S259 by dosage analysis (fig 1D). FISH indicated hemizygosity at these loci in the remaining parents. Three parents had mild learning disabilities but no history of congenital heart disease, neonatal hypocalcaemia, immune deficiency, or palatal abnormalities (fig $1 \mathrm{~A}-\mathrm{C}$ ). In addition to a learning disability, the mother of the fourth proband with DGS had a cleft palate (fig 1D). The parent of the child with VCFS also carries the diagnosis of VCFS; she has a cleft palate, learning disorder, and facial dysmorphism consistent with VCFS (fig 1E).

\section{PRENATAL STUDIES}

\section{Case 1}

DNA was obtained from the cultured amniocytes from the pregnancy at $50 \%$ risk for VCFS and the associated 22q11 deletion. Dosage analysis showed the presence of two copies of D22S75 (N25) in contrast to a single copy in both the affected mother and her affected daughter. FISH with an N25 specific cosmid confirmed the presence of two alleles in the fetus. Hence, the fetus did not appear to have inherited the maternal chromosome 22 bearing the deletion.

Fig 2A shows an example of metaphase chromosomes from the mother with VCFS after hybridisation with $\mathrm{N} 25$ and cos 82 cosmids. The chromosomes 22 are identified with $\cos 82$ marking the distal long arm of both homologues. A hybridisation signal for N25 is visualised on only one of the chromosomes 22 , consistent with a deletion. In contrast, in fig $2 \mathrm{~B}$, signals for both cosmids are seen on both chromosomes 22 of the at risk fetus. In addition to molecular studies, we recommended that a level II ultrasound examination and fetal echocardiogram be performed during the pregnancy to rule out a cleft palate and a congenital heart defect in the fetus. There were no apparent congenital anomalies seen on ultrasonography. The mother declined to have an echocardiogram. She was delivered of a normal appearing liveborn infant by spontaneous vaginal delivery at 36 weeks' gestation. There was no evidence of a palatal abnormality or facial dysmorphism. Dosage studies on DNA obtained from a sample of cord blood as well as FISH of peripheral blood lymphocytes confirmed the absence of a $22 \mathrm{q} 11$ deletion in the infant.

\section{Case 2}

FISH studies of metaphase chromosomes from the chorionic villus sample showed two copies of both D22S75 and D22S259. This finding was in contrast to results obtained in a 


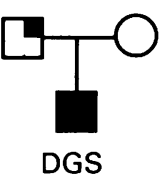

D

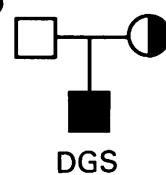

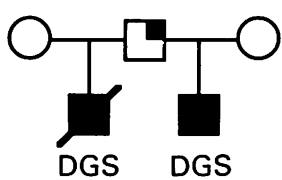

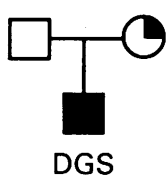

study of the affected sib in whom we had previously shown a deletion of both loci. The pregnancy is continuing.

\section{Discussion}

Initial studies by our laboratory and others reported an association between DGS, VCFS, and 22 q11 deletions. ${ }^{151619}$ In these studies over $90 \%$ of the patients studied had either cytogenetically visible interstitial deletions or submicroscopic deletions of $22 \mathrm{q} 11$. We have examined an additional 76 patients with either DGS or VCFS to determine the prevalence of $22 \mathrm{q} 11$ deletions in these disorders. In the present study, $83 \%$ ( 30 of 36 ) of presumed DGS patients and $68 \%$ (27 of 40 ) of putative VCFS patients have $22 \mathrm{q} 11$ deletions. If we include the 14 DGS and 14 VCFS patients we previously reported, then deletions were detected in $88 \%$ of patients referred with a diagnosis of DGS and $76 \%$ with VCFS.

The percentage of affected patients with a
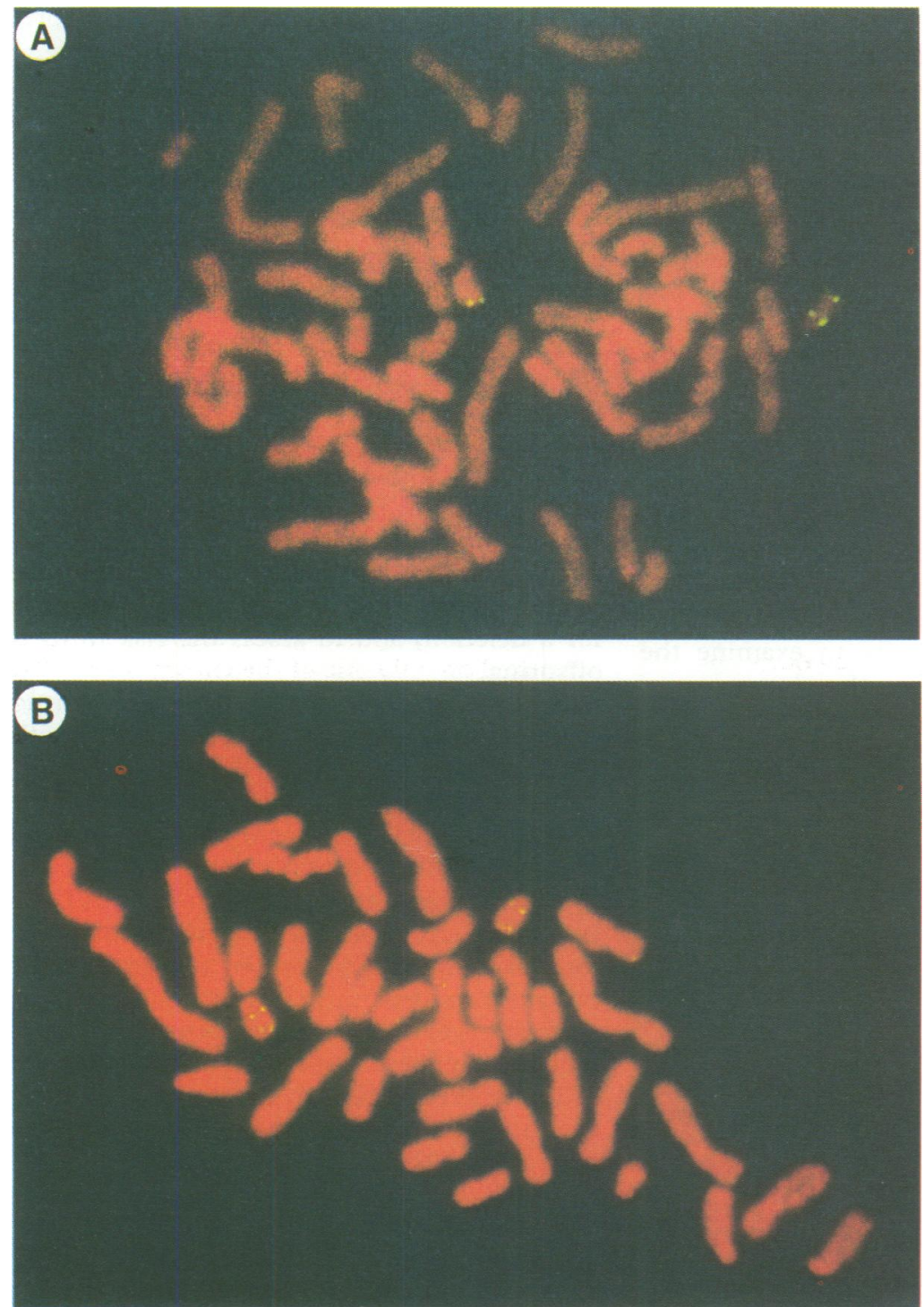

Figure 2 Fluorescence in situ hybridisation with chromosome 22 cosmids. The cosmids were labelled with biotinylated-11-dUTP and visualised with fuoresceinated avidin. The chromosomes 22 are identified with cos 82 marking the distal long arm and the test probe is a cosmid for N25, from the DGCR. (A) Metaphase chromosomes from mother affected with VCFS hybridised with cosmids cos 82 and N25. A hybridisation signal for N25 is seen on only one of the chromosomes 22 which is consistent with a deletion. (B) Hybridisation of cos82 and N25 to metaphase chromosomes from her at risk fetus. A hybridisation signal for N25 is present on each chromosome 22

$22 \mathrm{q} 11$ deletion is less than previously reported. If patients are included in the present study who do not have either DGS or VCFS, we may have underestimated the true prevalence of $22 \mathrm{q} 11$ deletions in these two groups of patients. It is of interest that the deletion appears to be more frequently detected in patients referred with the diagnosis of DGS. This may reflect the clinician's ability to diagnose DGS accurately in contrast to their ability to recognise VCFS.

The diagnosis of DGS is based on the presence of three findings: a congenital heart defect, hypocalcaemia, and a small or absent thymus. The clinical criteria for establishing a diagnosis of VCFS are not as restricted and it is likely that this study included patients in whom the diagnosis of VCFS was suspected but might not be corroborated. However, we relied on the clinical impressions of the referring geneticists and so the reported frequency of deletions in the population we present in this report may reflect the actual likelihood of detecting a deletion in clinical practice. The high frequency with which deletions were initially detected may reflect the small number of patients studied or the patient sample. The initial sample was derived from fewer clinicians and based on stricter inclusion criteria. Therefore, this study, which examines a large number of patients referred from numerous practising clinicians, provides us with a better estimate of the prevalence of 22q11 deletions in patients suspected to have DGS and VCFS.

The majority of patients appear to be deleted for the entire DiGeorge critical region (DGCR). We have not excluded the possibility that the non-deletion patients may have smaller deletions within the DGCR. Studies are in progress to determine whether these patients have deletions internal to the two markers (N25 and R32) used in this study. These disorders may also result from point mutations within critical genes in this region. As genes are identified, the non-deleted patients will be studied for the presence of point mutations. 
Alternatively, another locus may be implicated, such as a locus on $10 \mathrm{p}$. There have been several reports of $10 \mathrm{p}$ monosomy in association with DGS. ${ }^{625}$ Recently, molecular analysis of two DGS patients with cytogenetic deletions of $10 p$ did not show a deletion of $22 q 11$, suggesting that another locus is involved in the pathogenesis of DGS (unpublished results). Teratogens, such as retinoic acid and alcohol, and maternal diabetes have been associated with the DiGeorge anomaly. ${ }^{1}$ Molecular studies by our laboratory failed to detect a 22q11 microdeletion in two infants with DGS born to insulin dependent mothers, supporting the hypothesis that DGS is causally heterogeneous and that this represents a phenocopy of the $22 \mathrm{q} 11$ microdeletion syndrome. ${ }^{26}$

Approximately $8 \%$ of the patients studied showed familial transmission of the $22 \mathrm{q} 11 \mathrm{de}-$ letion. Deletions were detected in seven parents of patients with 22q11 deletions, five in this study and two in our previous report. ${ }^{19}$ Three parents were also diagnosed as having VCFS. However, three parents had only a learning disability or mild mental retardation and were only ascertained after the birth of an affected child. Such a mild phenotype may reflect which genes in 22q11 are deleted. As yet we have not detected a difference in the size of the deletion between numerous affected parents and their affected offspring. However, now that additional probes are available within and flanking the DGCR in $22 \mathrm{q} 11$, studies are in progress to identify the deletion boundaries in these families to determine if changes in the size of the deletion during meiosis accounts for the observed phenotypic differences. Phenotypic variability may also be explained by parent of origin of the deletion chromosome, genetic background, or in utero environment. We have recently identified highly polymorphic short tandem repeat polymorphisms in the DGCR which will enable us easily to examine the effect of parent of origin on the phenotype.

Recent studies suggest that several cytogenetically based disorders, such as MillerDieker and Wolf-Hirschhorn syndromes are better assessed for the presence of a microdeletion or cryptic translocation by FISH rather than routine cytogenetic analysis. ${ }^{27-29}$ We have established a FISH assay using cosmids for loci in the DGCR and have successfully used this assay to detect deletions in DGS and VCFS patients. ${ }^{30}$ Microdeletions of 22q11 have been detected in a total of 16 patients using FISH with cosmids specific for N25 and R32. FISH confirmed the results of dosage analysis in 10 patients, two with DGS and eight with VCFS. This highly sensitive assay eliminates the need for high resolution cytogenetic analysis to detect interstitial deletions of $22 \mathrm{q} 11$ in patients at high risk for a deletion.

In addition, we have shown the usefulness of FISH for prenatal testing for the detection of $22 \mathrm{q} 11$ deletions. FISH can be performed both on cultured amniocytes and chorionic villi. It does not require DNA extraction and time consuming Southern blotting necessary for dosage analysis. Hence, results are available faster. Traditionally, prenatal diagnosis of DGS and VCFS relied on ultrasonography and fetal echocardiography during the second trimester to detect the presence of a cleft palate or congenital heart defect. Detection of an affected fetus is limited by the ability to detect one of these features sonographically and, since the clinical features are variable, a normal sonogram would not definitively exclude the diagnosis of DGS or VCFS.

Patients with a 22q11 microdeletion who have a $50 \%$ risk of transmitting the deletion to their offspring can now be offered FISH for the prenatal detection of a $22 \mathrm{q} 11$ deletion as early as 10 to 12 weeks' gestation by chorionic villus sampling (CVS). Although normal parents of offspring with a de novo deletion are presumably at a low risk for a recurrence in subsequent pregnancies, we have not excluded the small possibility of germline mosaicism for a 22q11 deletion in these persons. In the absence of studies to dispute this possibility, these families might consider prenatal testing for reassurance, as did the second family presented in this report.

We had previously suggested that molecular and cytogenetic evaluation of the fetus for a 22q11 microdeletion should be offered to the parents when a conotruncal heart malformation is detected prenatally. ${ }^{31}$ Recent studies have shown that 22q11 microdeletions occur in 20 to $30 \%$ of newborns with non-familial, isolated conotruncal cardiac malformations, including truncus arteriosus, interrupted aortic arch, and tetralogy of Fallot. ${ }^{3233}$ These findings support our previous recommendation. These findings also suggest that, in addition to subjects with clinical features diagnostic of DGS and VCFS, persons with conotruncal heart defects need to be screened for $22 \mathrm{q} 11$ microdeletions to determine the aetiology of the disorder, identify other family members at risk for a deletion, and to assess the risk to their offspring.

Subjects with a $22 \mathrm{q} 11$ deletion have a $50 \%$ risk of transmitting the deletion to their offspring and should be offered genetic counselling. Furthermore, since the phenotype of the $22 \mathrm{q} 11$ microdeletion syndromes is variable, the fetus with a deletion is at risk for the spectrum of malformations seen in both DGS and VCFS. At present we are unable to predict the clinical outcome based on a deletion of the two markers used in the present studies. The correlation between the genotype and phenotype will require a detailed molecular analysis of the deleted region to determine which region or genes specify individual features of the phenotype.

The authors wish to thank Dr J Biegel for invaluable assistance with laboratory handling of patient specimens. We also thank G Lech and $M$ Dunn for their technical assistance. This work was supported in part by NIH grants HD26979 and CA39926 and March of Dimes Birth Defects Foundation Basic Research grant 1-FY91-106.

1 Lammer EJ, Opitz JM. The DiGeorge anomaly as a de- 
velopmental field defect. Am $\mathcal{F}$ Med Genet 1986;29:11327.

2 Back E, Stier R, Bohsen N, Adlung A, Hameister H. Partial monosomy 22pter $\rightarrow 11$ in a newborn with the clinical features of trisomy 13 syndrome. Ann Genet (Paris) 1980;23:244-88.

3 De la Chapelle A, Herva R, Koivisto M, Aula O. A deletion in chromosome 22 can cause DiGeorge syndrome. Hum Genet $1981 ; 57: 253-6$

4 Kelley RI, Zackai EH, Emanuel BS, Kistenmacher M, Greenberg $F$, Punnett $H$. The association of the DiGeorge anomalad with partial monosomy of chromosome 22. F Pediatr 1982;101:197-200.

5 Greenberg F, Crowder WE, Paschall V, Colon-Linares JC, Lubianski B, Ledbetter DH. Familial DiGeorge syndrome and associated partial monosomy of chromosome 22. Hum Genet 1984;65:317-19.

6 Greenberg F, Elder FFB, Haffner P, Northrup H, Ledbetter $\mathrm{DH}$. Cytogenetic findings in a prospective series of patients with DiGeorge anomaly. Am $\mathcal{f}$ Hum Genet 1988;43:605-11.

7 Augusseau S, Jouk S, Jalbert P, Prieur M. DiGeorge syndrome and 22q11 rearrangements. Hum Genet syndrome

8 Bowen P, Pabst H, Berry D, Collins-Nakai R, Hoo JJ. Thymic deficiency in an infant with a chromosome $\mathrm{t}(18 ; 22) \mathrm{t}(\mathrm{q} 12.2 ; \mathrm{p} 11.2)$ pat arrangement. Clin Genet 1986;29:174-7.

9 El-Fouly MH, Higgins JV, Kapur S, Matisoff DN, CostaFox $\mathrm{M}$. DiGeorge sequence in an infant with deletion of chromosome 22 and $\operatorname{dup}(9 \mathrm{p})$ due to adjacent type II disjunction. Am $\mathcal{F}$ Med Genet 1991;38:569-78.

10 Faed MJW, Robertson J, Swanson Beck J, Carter JI, Bose B, Madlon MM. Features of DiGeorge syndrome in a child with $45, \mathrm{XX}, 3,-22+\operatorname{der}(3), \mathrm{t}(3 ; 22)(\mathrm{p} 25 ; \mathrm{q} 11)$, Med Genet 1987;24:225-34.

11 Pivnick EK, Wilroy RS, Summit JB, Tucker B, Herro JG, Tharapel AT. Adjacent-2 disjunction of a maternal $t(9 ; 22)$ leading to duplication 9 pter $\rightarrow \mathrm{q} 22$ and deficiency of $22 \mathrm{pter} \rightarrow \mathrm{q} 11.2$. Am $₹$ Med Genet $1990 ; 37: 92-6$.

12 Wilson DI, Cross IE, Goodship JA, et al. A prospective cytogenetic study of 36 cases of DiGeorge syndrome. Am f Hum Genet 1992;51:957-63.

13 Schmickel RD. Contiguous gene syndromes: a component of recognizable syndromes. $\mathcal{F}$ Pediatr 1986;109:231-41.

14 Fibison WJ, Budarf M, McDermid H, Greenberg F, Emanuel BS. Molecular studies of DiGeorge syndrome. $A m \mathcal{F}$ Hum Genet 1990;46:888-95.

15 Driscoll DA, Budarf ML, Emanuel BS. A genetic etiology for DiGeorge syndrome: consistent deletions and microdeletions of 22q11. Am f Hum Genet 1992;50:924-33.

16 Scambler PJ, Carey AH, Wyse RKH, et al. Microdeletions within 22q11 associated with sporadic and familial DiGeorge syndrome. Genomics 119;10:201-6.

17 Carey AH, Kelly D, Halford S, et al. Molecular genetic study of the frequency of monosomy 22q11 in DiGeorge study of the frequency of monosomy 22q11

18 Emanuel BS, Driscoll D, Goldmuntz E, et al. Molecular and phenotypic analysis of the chromosome 22 microdeletion syndromes. In: Phenotypic analysis of Down syndrome and other aneuploid conditions. New York: Wiley Liss (in
press).

19 Driscoll DA, Spinner NB, Budarf ML, et al. Deletions and microdeletions of $22 \mathrm{q} 11.2$ in velo-cardio-facial syndrome. Am $\mathcal{F}$ Med Genet 1992;44:261-8.

20 Kelly D, Goldberg R, Wilson D, et al. Confirmation that the velo-cardio-facial syndrome is associated with haploinsufficiency of genes at chromosome 22. Am f Med Genet 1993;45:308-12.

21 Shprintzen RJ, Goldberg RB, Lewin ML, et al. A new syndrome involving cleft palate, cardiac anomalies, typical facies, and learning disabilities: velo-cardio-facial syndrome. Cleft Palate f 1978;15:56-62.

22 Shprintzen RJ, Goldberg RB, Young D, Wolford L. The velo-cardio-facial syndrome: a clinical and genetic analysis. Pediatrics 1981:67:167-72.

23 Williams MA, Shprintzen RJ, Goldberg RB. Male-to-male transmission of the velo-cardio-facial syndrome: a case report and review of 60 cases. $f$ Craniofac Genet 1985;5:175-80.

24 Shprintzen RJ, Wang F, Goldberg R, Marion R. The expanded velo-cardio-facial syndrome: additional
features of the most common clefting syndrome. $A m \mathcal{F}$ Hum Genet 1985;37:A77.
Hures of the most com

25 Monaco G, Pignata C, Rossi E, Mascellaro O, Cocozza S, Ciccimarra F. DiGeorge anomaly associated with $10 \mathrm{p}$ deletion. Am $\mathcal{f}$ Med Genet 1991;39:215-16.

26 Wilson TA, Blethen SL, Vallone A, et al. The DiGeorge anomaly with renal agenesis in infants of mothers with diabetes. Am $\mathcal{F}$ Med Genet (in press).

27 Kuwano A, Ledbetter SA, Dobyns WB, Emanuel BS, Ledbetter DH. Detection of deletions and cryptic translocations in Miller-Dieker syndrome by in situ hybridization. Am ₹ Hum Genet 1991;49:707-14.

28 Altherr MR, Bengtsson U, Elder FFB, et al. Molecular confirmation of Wolf-Hirschhorn syndrome with a subtle confirmation of Wolf-Hirschhorn syndrome with a

29 Goodship J, Curtis A, Cross I, et al. A submicroscopic translocation, $t(4 ; 10)$, responsible for recurrent WolfHirschhorn syndrome identified by allele loss and fluorescent in situ hybridisation. F Med Genet 1992;29:451-4.

30 Emanuel BS, Budarf ML, Sellinger B, Goldmuntz E, Driscoll DA. Detection of microdeletions of 22q11.2 with fluorescence in situ hybridization (FISH): diagnosis of DiGeorge syndrome (DGS), velo-cardio-facial (VCF) syndrome, CHARGE association and conotruncal cardiac malformations. Am $\mathcal{F}$ Hum Genet 1992;51:A3.

31 Driscoll DA, Budarf ML, Emanuel BS. Antenatal diagnosis of DiGeorge syndrome. Lancet 1991;338:1390-1.

32 Goldmuntz E, Driscoll DA, Emanuel BS. Microdeletions of chromosome 22 in patients with conotruncal cardiac defects. Am 7 Cardiol 1992;70:557.

33 Goldmuntz E, Driscoll D, Budarf ML, et al. Microdeletions of chromosomal region $22 \mathrm{q} 11$ in patients with congenital conotruncal cardiac defects. $\mathcal{f}$ Med Genet 1993;30:807-12. 